\title{
Maternal sympathetic stress impairs follicular development and puberty of the offspring
}

\author{
Rafael Barra ${ }^{1}$, Gonzalo Cruz ${ }^{1,2}$, Artur Mayerhofer ${ }^{3}$, Alfonso Paredes ${ }^{1}$ and Hernán E Lara ${ }^{1}$ \\ ${ }^{1}$ Laboratory of Neurobiochemistry, Department of Biochemistry and Molecular Biology, Faculty of Chemistry and \\ Pharmaceutical Sciences, Universidad de Chile, 8380492 Independencia, Santiago Chile, ${ }^{2}$ Departamento de \\ Fisiología, Facultad de Ciencias, Centro de Neurobiología y Plasticidad Cerebral (CNPC), Universidad de Valparaíso, \\ 2360094 Valparaíso, Chile and ${ }^{3}$ Anatomy III - Cell Biology, Ludwig Maximilian University, Schillerstrasse 42 \\ D-80336 Munich, Germany
}

Correspondence should be addressed to H E Lara; Email: hlara@ciq.uchile.cl

\begin{abstract}
Chronic cold stress applied to adult rats activates ovarian sympathetic innervation and develops polycystic ovary (PCO) phenotype. The PCO syndrome in humans originates during early development and is expressed before or during puberty, which suggests that the condition derived from in utero exposure to neural- or metabolic-derived insults. We studied the effects of maternal sympathetic stress on the ovarian follicular development and on the onset of puberty of female offspring. Timed pregnant rats were exposed to chronic cold stress $\left(4^{\circ} \mathrm{C}, 3 \mathrm{~h}\right.$ /daily from 1000 to $1300 \mathrm{~h}$ ) during the entire pregnancy. Neonatal rats exposed to sympathetic stress during gestation had a lower number of primary, primordial, and secondary follicles in the ovary and a lower recruitment of primary and secondary follicles derived from the primordial follicular pool. The expression of the FSH receptor and response of the neonatal ovary to FSH were reduced. A decrease in nerve growth factor (NGF) mRNA was found without change in the low-affinity NGF receptor. The FSH-induced development of secondary follicles was decreased. At puberty, estradiol plasma levels decreased without changes in LH plasma levels. Puberty onset (as shown by the vaginal opening) was delayed. Ovarian norepinephrine (NE) was reduced; there was no change in its metabolite, 3-methoxy-4-hydroxyphenylglycol, in stressed rats and no change in NE turnover. The changes in ovarian NE in prepubertal rats stressed during gestation could represent a lower development of sympathetic nerves as a compensatory response to the chronically increased NE levels during gestation and hence participate in delaying reproductive performance in the rat.

Reproduction (2014) 148 137-145
\end{abstract}

\section{Introduction}

Stress as an adaptive response of the body produces a wide range of biochemical and behavioral manifestations to respond to a threat (Chrousos 1998, Szabo 1998, Tache \& Brunnhuber 2008). This apparently beneficial response of the body can become a problem when the stress becomes chronic. (Goldstein \& Kopin 2008). Cold stress increases the noradrenergic tone in the rat, without producing changes in serum epinephrine or adrenocorticotropic hormone levels (Pacak et al. $1998 a, b)$. We previously used this stress paradigm and demonstrated that when applied to adult rats it increased ovarian norepinephrine (NE) levels and induced ovarian function alterations, which led to polycystic ovarian morphology in rats (Paredes et al. 1998, Dorfman et al. 2003, Greiner et al. 2005). Polycystic ovary is the most common ovarian disease in women during their reproductive life (Fauser et al. 2012), is expressed early in development, thus proposing the likelihood of fetal origin.
The preferential sympathetic component of the chronic cold stress paradigm permits one to study the neural noradrenergic component of the physiological response (Goldstein \& Kopin 2008) and the factors regulating the adequate establishment of the sympathetic innervation, such as nerve growth factor $(N G F)$, by the respective innervated organ, including the ovary (Levi-Montalcini 1987).

The two important emerging questions are what are the implications of stress during gestation life and whether and how stress may affect the predisposition for, or programing of adult disease (Glover et al. 2010, Nugent et al. 2012, Rinaudo \& Wang 2012), Alteration of reproductive function by early exposure to stress would compromise the survival of the species. Furthermore, the impact of prenatal programing of adult disease can be transmitted to future generations. In this regard, pre-eclampsia is characterized by high sympathetic activity that affects blood pressure in gestating mothers and induces changes that affect the progeny (Schobel et al. 1996, Takiuti et al. 2003), presumably through 
changes in the availability of NE to the fetal environment. Thus, newborn babies have changes in weight and metabolic parameters.

To understand the risk of chronically enhanced sympathetic nerve activity to the in utero development, we studied whether chronic cold stress (as a direct sympathetic stimulus) applied to rats during the entire gestation period may induce alterations in ovarian function during postnatal development of the progeny and whether it may affect reproductive performance during adult life.

\section{Materials and methods Animals}

Sprague-Dawley rats weighing 250-300 g were maintained at $20{ }^{\circ} \mathrm{C}$ with a $12 \mathrm{~h}$ light: $12 \mathrm{~h}$ darkness cycle. Water and food were provided ad libitum. Estrual cycling activity of rats was monitored by vaginal smears. In the afternoon of proestrus, rats were mated and the next morning pregnancy was confirmed by checking a vaginal sperm plug. We randomized 23 pregnant rats into two groups of ten control rats and 13 stressed rats. The control rats were maintained at room temperature during the entire pregnancy and the stressed rats were moved to a cold room at $4{ }^{\circ} \mathrm{C}$ for $3 \mathrm{~h}$ /each day during the entire pregnancy. All experimental procedures were approved by the Bioethics Committee of the Faculty of Chemistry and Pharmaceutical Sciences at the Universidad de Chile and complied with national guidelines (CONICYT Guide for the Care and Use of Laboratory Animals). All efforts were made to minimize the number of animals used and their suffering.

\section{Experimental design}

After birth, the male rats were given to a foster mother and the female pups stayed with their mothers up to postnatal day 4 (PND4), at which time they were mixed and used for the experiments. The neonates used for prepubertal studies were maintained with their mothers up to 30 days old.

\section{Study in neonatal rats}

Four neonates from the control group and six neonates derived from cold-stressed dams were killed at PND4; the right ovary was fixed in Bouin's fixative and the left ovary was frozen at $-80{ }^{\circ} \mathrm{C}$. The ovaries of a second group of 4-day-old neonates ( $n=8$ control and ten stressed rats) were immediately frozen and stored at $-80^{\circ} \mathrm{C}$. A third group of neonates $(n=16$ control and 22 stressed rats) was used to study the 'in vitro' effects of follicle-stimulating hormone (FSH) on the ovary.

\section{Study in 30-day-old prepubertal rats}

A group of nine control rats and 14 stressed prepubertal rats were killed at 30 days old; the right ovary was fixed in Bouin's fixative and the left ovary was frozen at $-80^{\circ} \mathrm{C}$. Plasma samples were collected and stored at $-20^{\circ} \mathrm{C}$ for steroid analysis.
Study of vaginal opening as index of puberty

A group of six control rats and nine stressed rats born from control and stressed dams, respectively, was followed to study the age of vaginal opening as an index of puberty. We analyzed the capacity of these rats to develop a cyclic estrual activity.

\section{Incubation assay}

Pairs of ovaries from stressed and control rats of 4 days old were incubated for $24 \mathrm{~h}$ at $37{ }^{\circ} \mathrm{C}$ with $95 \%$ oxygen and $5 \% \mathrm{CO}_{2}$, as described previously (Mayerhofer et al. 1997). Four experimental conditions were tested: control+incubation media, stress + incubation media, control $+\mathrm{FSH}$, and stress $+\mathrm{FSH}$. The incubation media utilized was DMEM/F12 Gibco (Invitrogen Corporation), supplemented with $100 \mathrm{U}$ of antibiotics (penicillin + streptomycin) and $0.5 \mathrm{mM} 3$-iso-4-butyl, methylxantine to inhibit phosphodiesterase. We used human FSH (F4021, $7000 \mathrm{IU} / \mathrm{mg}$, Sigma Chemical Co.) at a final concentration of $10 \mathrm{IU} / \mathrm{ml}$ incubation media.

After $24 \mathrm{~h}$ of incubation, the ovaries were fixed to perform morphometric analysis of follicles; the incubation media were frozen at $-80{ }^{\circ} \mathrm{C}$ for determination of CAMP.

\section{Morphometry}

Ovaries previously fixed were embedded in paraffin, cut into $6-\mu \mathrm{m}$ sections, and stained with hematoxylin and eosin. Morphometric analyses of whole ovaries were done as described previously (Cruz et al. 2012).

\section{Measurements of mRNA levels by real-time RT-PCR}

Total RNA from the ovaries was prepared using the Chomczynski \& Sacchi (1987) method of RNA extraction. The total RNA extracted was submitted to RT reaction to obtain cDNA. Then, cDNA obtained was amplified in real-time PCR equipment (IQ5 thermocycler, Bio-Rad Laboratories, Inc.) using specific primers for NGF, low-affinity NGF receptor $\left(p 75^{N T R}\right)$, and receptor for FSH $(F S H R)$ as described previously (Dissen et al. 2000, Romero et al. 2002). All of the samples were analyzed in triplicate.

\section{Determination of ovarian levels of $N E$, 3-methoxy-4-hydroxyphenylglycol by HPLC}

Ovaries previously stored at $-80{ }^{\circ} \mathrm{C}$ were weighed and then homogenized in perchloric acid, $0.25 \mathrm{M}$, and centrifuged at $12000 \mathrm{~g}$ for $10 \mathrm{~min}$ at $4{ }^{\circ} \mathrm{C}$. The resulting supernatant was filtered and used for the measurement of NA and 3-methoxy4-hydroxyphenylglycol (MHPG) using a HPLC system (Sotomayor-Zarate et al. 2008, 2011) as previously described.

\section{Determination of cAMP concentrations}

All cAMP concentrations were determined by EIA, using a cAMP EIA Kit (Cayman Chemical Co., Ann Arbor, MI, USA) in accordance with the manufacturer's instructions. The coefficient of variation was $<10 \%$ (Pradelles et al. 1989). 


\section{Determination of serum levels of estradiol}

Estradiol $\left(E_{2}\right)$ levels were determined using an enzyme immunoassay according to the manufacturer's instructions (Alpco Diagnostic, Windham, NH, USA). Intra- and inter-assay variations were $<5 \%$ for $E_{2}$. The minimal detectable value was $10 \mathrm{pg} / \mathrm{ml}$.

\section{Statistical analyses}

The data are expressed as the mean士s.E.M. values. To determine significant differences among multiple groups, we used one-way ANOVA followed by the Newman-Keuls post hoc test. To determine the differences between two groups, we used Student's $t$-test.

\section{Results}

\section{Effects of stress during gestation in early follicular development}

Early follicular development was assessed by morphometric analysis of all follicles of neonatal ovaries at PND4. Representative microphotographs of the ovaries are shown in Fig. 1. There were several differences between the ovaries from the control (Fig. 1A) and stressed (Fig. 1B) groups. First, the ovary of the control rats had a higher number of developing follicles than the ovary of stressed rats. Second, in the stressed rats (Fig. 1B), there was an increase in naked primordial follicles (arrows head), which are found primarily at the periphery of the ovary. Main differences were found in the number of healthy primordial follicles (Fig. 1A, black arrows), which have a healthy nuclei with a spherical shape compared with apoptotic follicles (Fig. 1B, arrow head), which present pycnotic nuclei with an irregular shape and some granulosa cells. The total number of follicles (including apoptotic follicles which are not more than 60 per ovary of stressed rats) was decreased in the offspring of rats stressed during gestation compared with the control rats (Fig. 1C). The more numerous follicles at this age are primordial follicles, also named 'follicular reserve pool', which were diminished in the stress group compared with the control group (Fig. 1D). This decrease is accompanied by an increase in apoptotic follicles undergoing the process of attrition (Fig. 1E) and thus total primordial pool (healthy + apoptotic) was unchanged. The primary and secondary follicles were also decreased in the stress group compared with the control group (Fig. 1F and G).

Neonatal follicular development
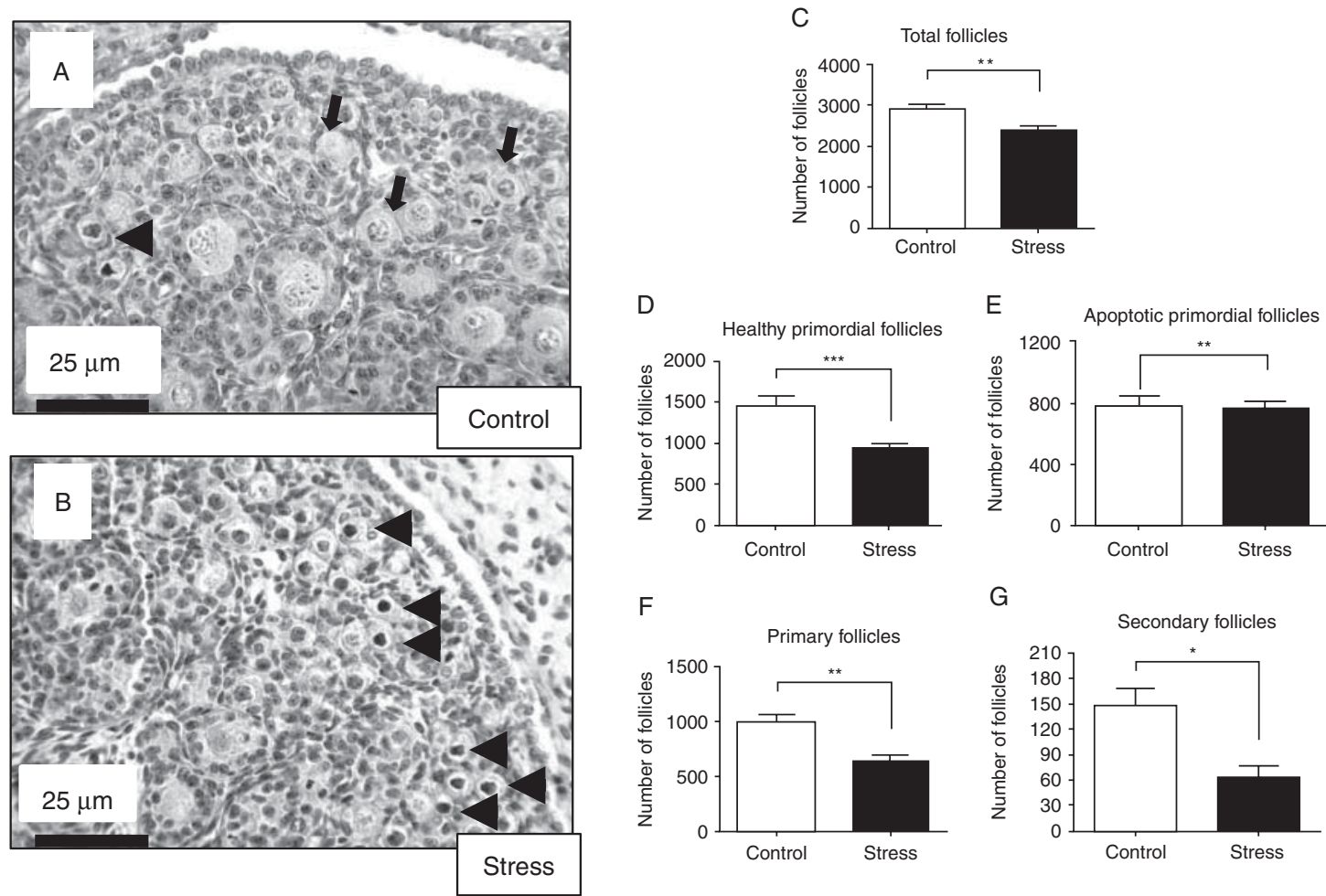

\footnotetext{
Figure 1 Representative pictures of neonatal ovaries from offspring rats from control (A) and stressed mothers (B). Black bar at the bottom of each picture corresponds to magnification of the picture. Arrows head represent apoptotic follicles. (C-G) Neonatal ovarian morphometry. (C) The total number of follicles in the control and stress groups; (D) healthy primordial follicles in the control and stress groups; (E) the number of atretic follicles in the control and stress groups; (F) the primary follicles in the control and stress groups; and (G) the secondary follicles in the control and stress groups. Results represent the mean \pm S.E.M. values of three rats for control and six rats for stress group. ${ }^{*} P<0.05$ vs control; ${ }^{* *} P<0.01$ vs control; and $* * * P<0.001$ vs control.
} 
In addition, the ratio between developing follicles (primary + secondary) and total healthy follicles (healthy primordial + primary + secondary) was decreased in the stress group compared with the control group (control group $39.30 \pm 2.91$ vs stress group $28.75 \pm 2.189$, $P<0.05$ vs control, $n=5$ in each condition), which suggests that in addition to a lower number of follicles there is a decreased passage from the primordial stage to additional stages of development.

\section{The effect of stress during gestation on the ovarian mRNA expression of NGF, $\mathrm{p} 75^{\mathrm{NTR}}$, and FSHR of the female progeny}

Early works (Dissen et al. 2001, 2009) describe the role of NGF, $p 75^{N T R}$, and NTRK1 (TRKA) in early follicular development and in the expression of FSHR. Thus to correlate the early changes in follicular development found in this study that are induced by a neurogenic stimulus such as sympathetic stress, we measured the expression of $N G F$ and its receptors in addition to its functional expression as the FSHR. The levels of mRNA for $N G F, p 75^{N T R}$, and FSHR obtained by qRT-PCR are shown in Fig. 2. The stress group presented a significant decrease in the levels of FSHR and NGF in the ovary (Fig. 2A and B). No differences were found in $p 75^{N T R}$ levels between the control and stress groups (Fig. 2C).

\section{The effect of stress during gestation on the neonatal ovarian responsiveness to $\mathrm{FSH}$ of the female progeny}

To understand the ovarian response to $\mathrm{FSH}$, we incubated PND4 ovaries for $24 \mathrm{~h}$ with FSH or with the incubation media. The number of secondary follicles after incubation with FSH was higher in the control rats than in the stress group (Fig. 3A). In addition, the secondary follicles reached a higher diameter in the control ovaries under FSH stimulation compared with the secondary follicles from stressed rats (Fig. 3B). In the stressed group, we observed a lower capacity of ovaries to produce cAMP under the incubation with $\mathrm{FSH}$, which strongly suggests that there is a decreased responsiveness of the ovary to $\mathrm{FSH}$ and thus to develop secondary follicles under a gonadotropic stimulation (Fig. 3C).

\section{The effect of stress during gestation on the age of vaginal opening and follicular development near to puberty in the female offspring}

To analyze follicular development during the prepubertal stage of development, we studied the ovaries of control and stressed rats at the age of 30 days to quantify antral follicular development by morphometry and to relate it with plasma $E_{2}$ levels. There was a decrease in the number of larger-size antral follicles preovulatory at 30 days old in the stress group (Fig. 4A). The stressed rats presented decreased plasma $\mathrm{E}_{2}$ levels (Fig. 4B).
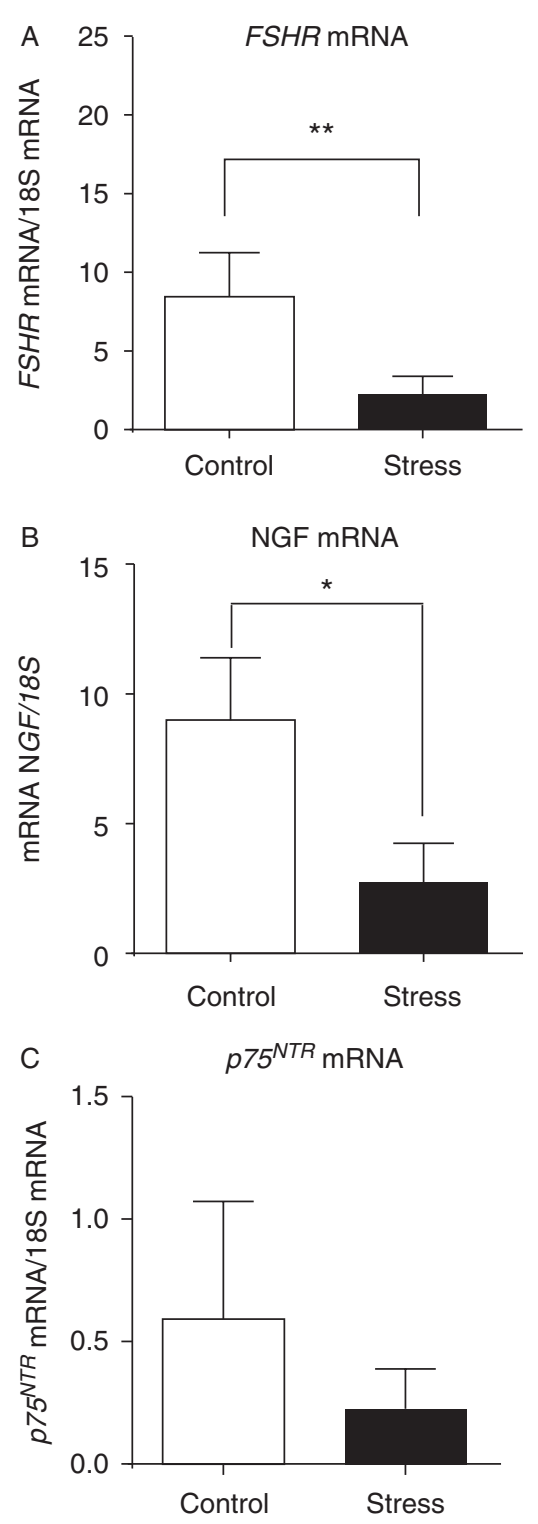

Figure 2 Quantification of the levels of mRNA to $F S H R(\mathrm{~A}), N G F(\mathrm{~B})$, and $p 75^{N T R}(\mathrm{C})$ by qRT-PCR. Results are expressed as the mean \pm s.E.M. values of four experiments in each condition. ${ }^{*} P \leq 0.05 ;{ }^{*} P<0.01$ vs control.

Another group of rats was monitored to determine at which stage they reached vaginal opening as an index of puberty. Rats exposed to stress during gestation had delayed puberty. Figure $4 \mathrm{C}$ demonstrates that $50 \%$ of rats reached vaginal opening by 32 days of age in the control group, whereas in the stress group, 50\% of rats reached vaginal opening at $\sim 35$ days of age.

\section{The effect of stress during gestation on ovarian NE and turnover of the female offspring}

Previous evidence showed that extrinsic noradrenergic innervation develops just before puberty in the rat, as demonstrated by an increase in the concentration of $\mathrm{NE}$ 

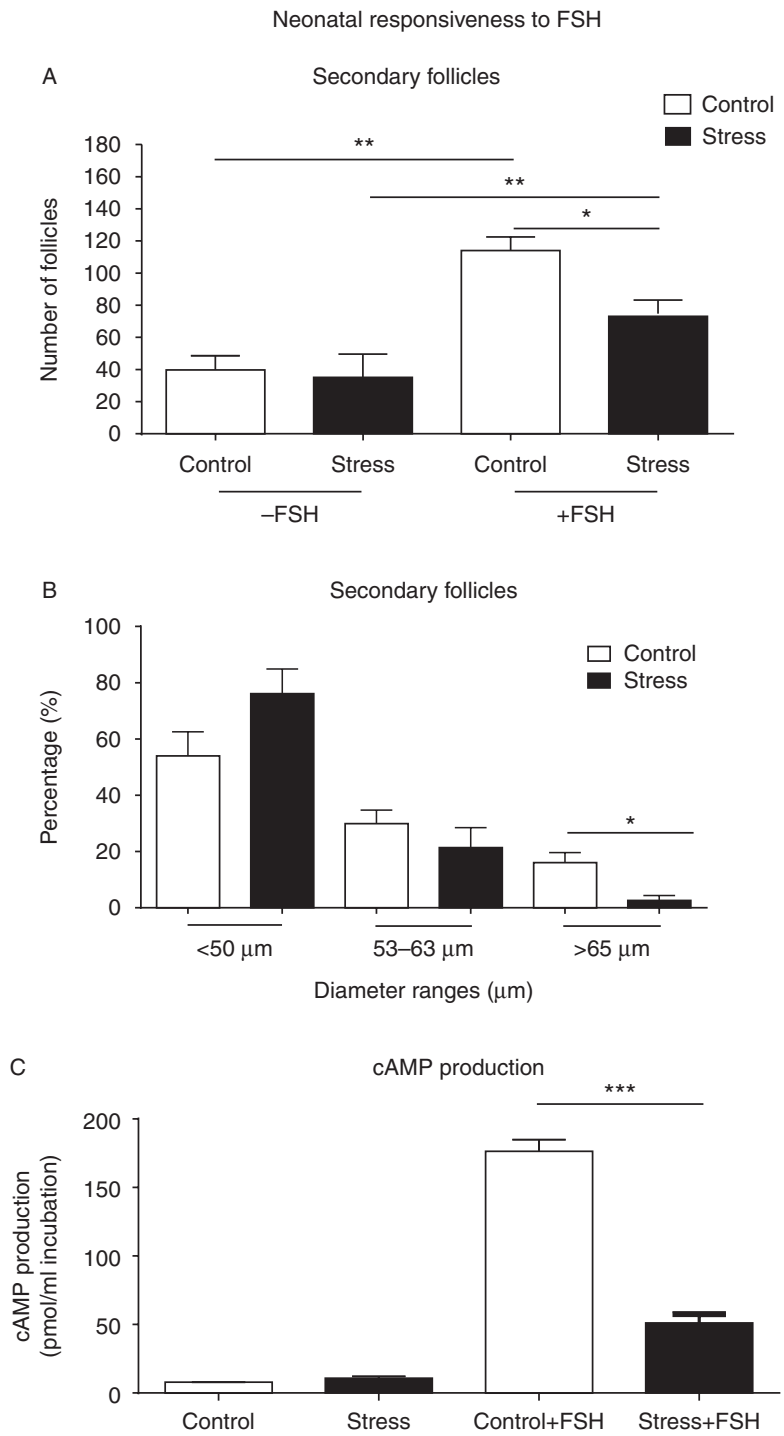

Figure 3 Responsiveness of the neonatal (4 days old) ovary to in vitro incubation with FSH (A) shows the presence of secondary follicles after $24 \mathrm{~h}$; (B) a morphometric analysis of the secondary follicles; and (C) the concentration of CAMP determined in the incubation media. Results are expressed as the mean \pm S.E.M. values of four experiments in each condition. ${ }^{*} P \leq 0.05$; ${ }^{* *} P<0.01$; and ${ }^{* * *} P<0.001$ vs control.

and in the induced release of NE from the tissue (Ricu et al. 2008). At PND30, there was a decrease in the concentration of $\mathrm{NE}$ in the progeny of rats stressed during gestation compared with the progeny of the control group (Fig. 4D). This decrease in NE was followed by a similar decrease in its metabolite MHPG (Fig. 4E); thus, the turnover rate was similar (Fig. 4F).

\section{The effect of stress during gestation in the acquisition of regular cycling activity in the offspring}

Vaginal cytology analysis is commonly used to determine the presence of ovulatory or anovulatory cycles. Approximately, $80 \%$ of the control rats reached normal cycling activity after vaginal opening (Fig. 5A) compared with fewer than $40 \%$ of the offspring of the mothers stressed during gestation reached normal cycling activity at puberty (Fig. 5B). The total number of cycles after the first month of observation was significantly decreased in the stressed rats (Fig. 5C); this effect was preferentially found during the first two weeks of observation (Fig. 5D). No differences were found during the last two weeks of observation (Fig. 5E).

\section{Discussion}

There is ample evidence linking an exposure to stress during gestation with harmful effects observed in the offspring. Different types of gestational stressors have been identified to program adult disease (Glover et al. 2010, Rinaudo \& Wang 2012). In this study, we found that chronic prenatal sympathetic stress during gestation decreases the follicular reserve pool and impairs early follicular development during neonatal development in the offspring and the acquisition of female reproductive capacity and most likely the end of reproductive life.

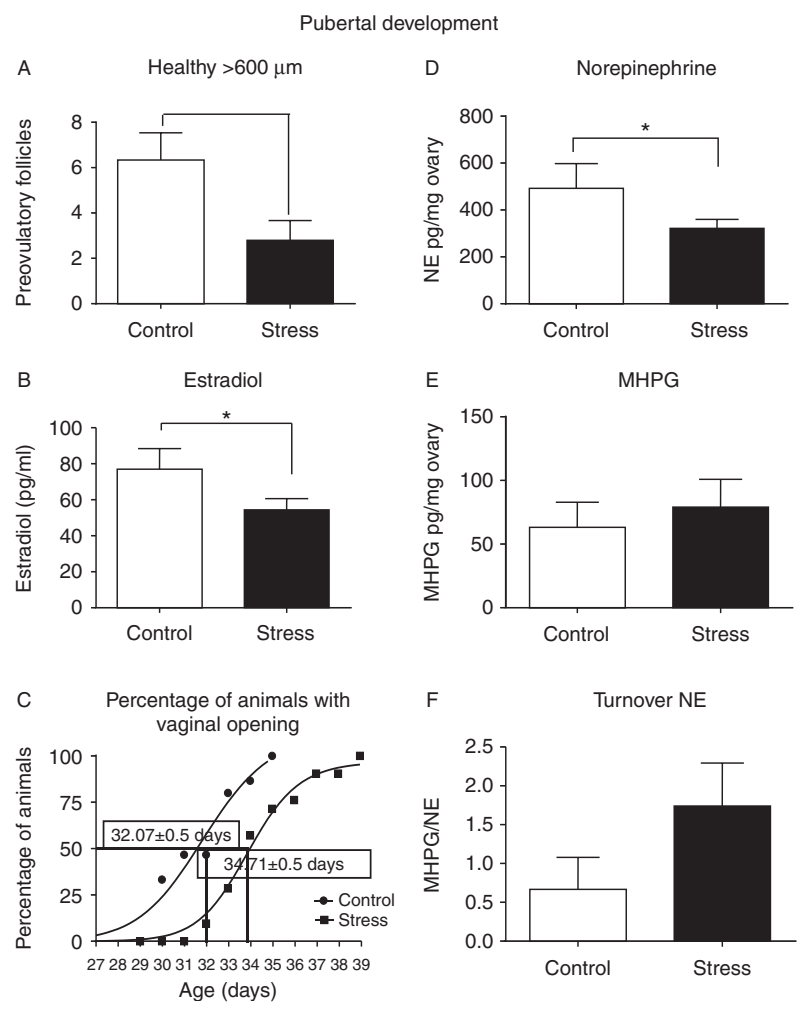

Figure 4 Effect of gestational stress in the number of antral preovulatory follicles $(A)$, in the plasma concentration of estradiol $\left(E_{2}\right)(B)$, and in the age of vaginal opening $(C)$. Data for follicles and $E_{2}$ correspond to the mean \pm S.E.M. values of four and six individual animals, respectively, at the age of 30 days. ${ }^{*} P<0.05$ vs control. In D-F are shown the norepinephrine (NE) concentration and turnover. (D) ovarian NE, (E) 3-methoxy-4-hydroxyphenylglycol (MHPG) concentration, and (F) the turnover ratio. Results correspond to the mean \pm S.E.M. values of four rats for control and seven for gestational stressed rats. $* P<0.05$ vs control. 
Estrous cycling activity

A

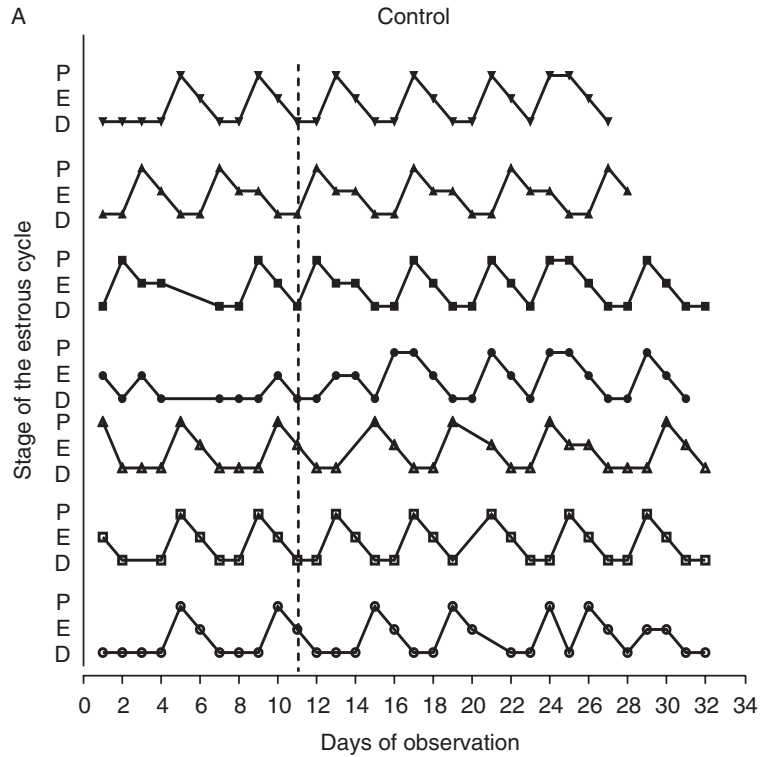

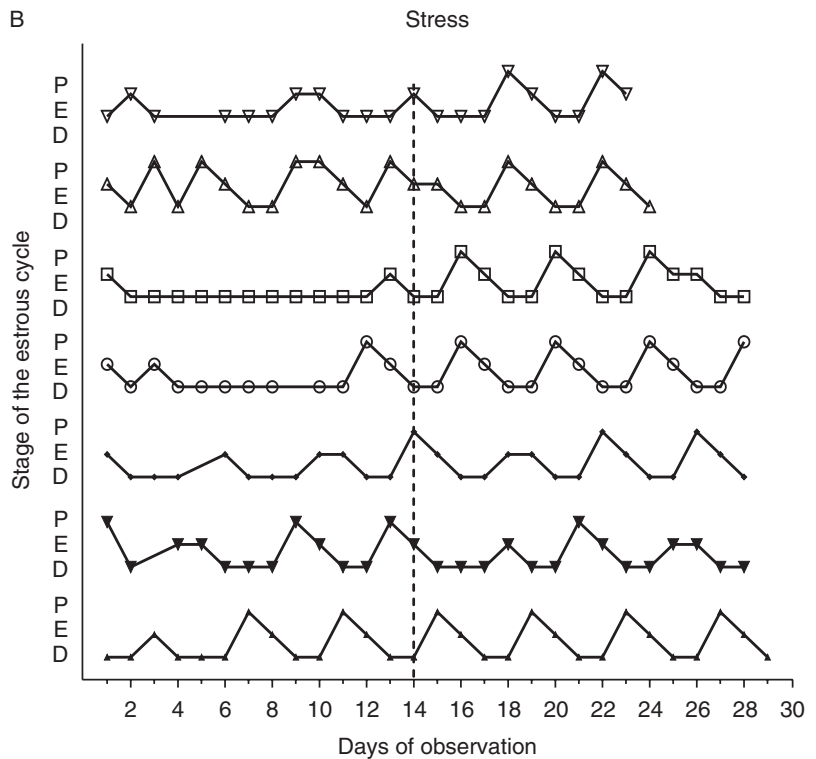

C

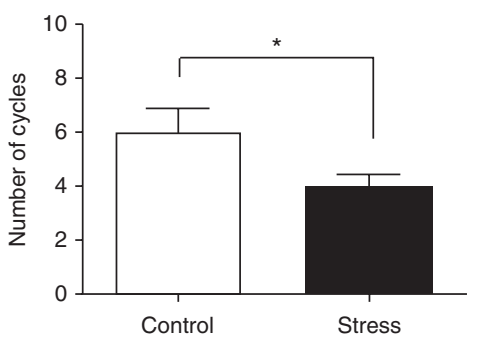

$\mathrm{D}$

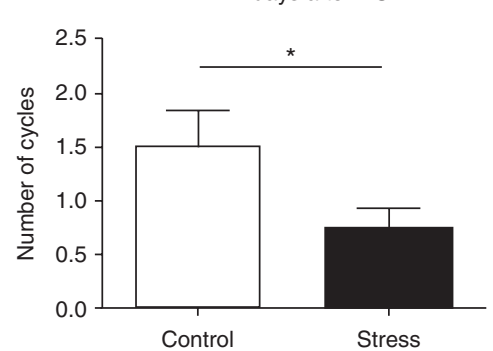

$\mathrm{E}$

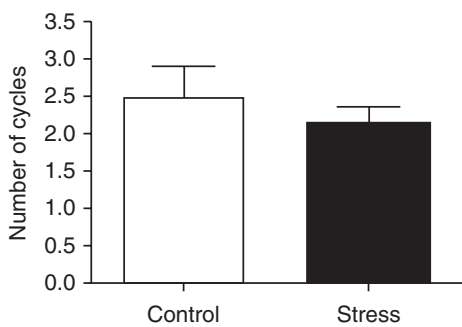

Figure 5 Gestational stress and its consequence on the estrual cycling activity after vaginal opening. Results in (A and B) represent data for representative animals for each group and day 0 corresponds to the first day after rats presented vaginal opening. Rats were observed for 4 weeks. The period of observation was divided in two groups. (C) The total number of cycles during the 4-week period. (D and E) Correspond to the estrual cycling activity during the first 2 weeks (D) and during the following 2 weeks (E). Results correspond to 12 control and 12 rats with gestational stress and represent the mean \pm s.E.M. values. ${ }^{*} P<0.05$ vs control.

\section{Stress during gestation decreases the follicular reserve pool}

To the best of our knowledge, there is no previous evidence regarding prenatal stress exposure and its effect on early ovarian function. The similar number of total primordial follicles (healthy + apoptotic) in the stress group compared with the control group indicates that both groups of rats likely presented with similar amounts of oocytes before follicular assembly occurred. The decreased number of healthy primordial follicles and the increased number of oocytes undergoing apoptosis (apoptotic follicles) at PND4 indicate a likely failure during the follicular assembly process in animals under gestational stress. The bulk of primordial follicle assembly occurs within days 1-4 after birth and the process is commanded by paracrine regulators, such as NGF and others (Dissen et al. 2001, Pepling 2012). The increased number of apoptotic follicles at PND4 could be the result of the decreased expression of NGF.
This neurotrophin supports oocyte survival and favors proliferation and differentiation of granulosa cells in follicles at different stages of development (Dissen et al. 1995, 2000, 2009). Interestingly, the NGF knockout mice have a similar follicular distribution of primordial follicles as the one observed in this study, i.e. a decrease in healthy follicles and an increase in nonassembled follicles that strongly support our finding (Dissen et al. 2001).

\section{Stress during gestation impairs the initial recruitment of follicles and decreases the progression from primary to secondary follicles}

While the follicular assembly is occurring, some already assembled follicles begin to differentiate in primary follicles and subsequently in secondary follicles (Pepling 2012). At PND4, the primordial, primary, and secondary follicles develop in the ovary (Pepling 2012). The decreased number of primary follicles found in the stress 
group compared with the control group could be the product of a decreased number of healthy primordial follicles that result from a poor follicular assembly. However, the decreased ratio observed between developing follicles and total follicles (including primordial) indicates that independent of the number of primordial follicles, there is an alteration of the recruitment of follicles or an alteration in its control. NGF has been postulated to participate in the recruitment of follicles because the peptide is necessary for the differentiation of pregranulosa cells into granulosa cells (Dissen et al. 2009, Pepling 2012). In addition to this role, the incubation of ovaries with NGF enhances the development of primary follicles by increasing the expression of FSHR (Romero et al. 2002). The decrease in Ngfb mRNA found in this study could be paralleled with a similar decrease in the NGF peptide, as we previously demonstrated to occur in the adult rat neonatally treated with estradiol valerate, (EV) (Sotomayor-Zarate et al. 2008). The small size of the ovary made it impossible to get enough tissue for the EIA to measure NGF.

Hence, the decrease in NGF mRNA levels could be the cause of the decreased mRNA levels of FSHR that we observed in this study. The decreased FSHR levels can result from the slow trafficking from primary to secondary follicles, which finally leads to a poor amount of secondary follicles in the stressed group of rats.

\section{A loss of sensitivity to FSH is the cause of low development of secondary follicles in the offspring of rats stressed during gestation}

The expression of NGF is functionally coupled to the expression of FSHR (Romero et al. 2002); if there is a decreased expression of FSHR in early developing follicles, we should expect a decreased capacity of FSH to promote the transition from primary to secondary follicles and the growth of this type of follicles. To examine this decreased expression of FSHR, we incubated 4-day-old ovaries from the stress and control groups with FSH for $24 \mathrm{~h}$. The development of preantral follicles depends on several paracrine factors and on the extrinsic regulation performed by gonadotropins and innervations (Mayerhofer et al. 1997, Dissen et al. 2009, Sirotkin 2011). After $24 \mathrm{~h}$ of incubation with only the media, the number of secondary follicles was similar between control and stress groups, which suggest that an extrinsic factor is involved in the decreased development of secondary follicles observed in vivo in the stress group. Interestingly, the incubation with FSH for $24 \mathrm{~h}$ increases the development of secondary follicles in both groups; however, the number of secondary follicles in the stress group was lower compared with that in the control group. In addition, as FSH promotes the proliferation and differentiation of granulose cells (DeManno et al. 1999) leading secondary follicles to grow, the decrease in larger-size secondary follicles observed in the stress group is due to a lower sensitivity to $\mathrm{FSH}$. The low levels of cAMP reached after FSH stimulation in the stress group compared with the control group indicate that the lower sensitivity to $\mathrm{FSH}$ is due to a lower response of follicles because of low FSHR expression. Altogether, these results demonstrate that the lower response to $\mathrm{FSH}$ leads to a lower trafficking from primary to secondary follicles and to a lower development of secondary follicles in the offspring of rats submitted to sympathetic gestational stress.

\section{Slow trafficking of follicles may cause delayed puberty in the offspring of rats stressed during gestation}

The decreased number of follicles at all stages of development and the decreased sensitivity of preantral follicles to FSH in the stressed progeny suggest a condition persisting through pubertal development. The decreased number of larger-size antral follicles seen at 30 days old $(>600 \mu \mathrm{m})$, which corresponds to the first cohort of follicles that will ovulate, was a likely consequence of a lesser amount of follicles capable of responding to FSH during early development in the stress group. This type of follicles is the primary source for the increasing levels of $E_{2}$ before puberty, which produces the positive feedback on luteinizing hormone (LH). Coherently, we observed that prepubertal rats aged 30 day had lower levels of $E_{2}$.

\section{Programing effect of sympathetic stress}

We previously described that stress-induced sympathetic activation using a 4-week chronic cold stress to adult rats (Dorfman et al. 2003) increased NE and NGF concentrations in the ovary in a manner that is causally related to a centrally originated increase in sympathetic activity (Fiedler et al. 2006, Jara et al. 2010). The decreased ovarian NE concentration found in the ovary of prepubertal rats could be the result of a compensatory response to the chronic hyper-noradrenergic condition during gestation. The fact that there is a similar change in the metabolite MHPG explains the lack of change observed in the turnover rate of the sympathetic neurons, which could indicate that the decrease in NE results from poorly developed noradrenergic nerves. The immunoblockade of NGF during neonatal development in rats decreased NGF and blocked sympathetic nerve development (Lara et al. 1990a,b), thus the decreased NGF expression found at early stages of development could be the responsible of the low NE activity and probably nerve development. We also observed decreased follicular development, delayed puberty, and low $E_{2}$ plasma levels (Lara et al. 1990b). Similarly, the lower number and poor development of the larger-size follicles observed in this study were correlated with decreased serum levels of $E_{2}$ measured in stressed rats. 
Preovulatory follicles are the primary source of plasma levels of $E_{2}$. This first wave of follicles is necessary to reach the increasing levels of $E_{2}$ and to produce a feed-forward on release of $\mathrm{LHRH}$ and subsequently increasing release of LH that induces ovulation (Ebling 2005). This increase in $E_{2}$ levels produces changes in the vaginal epithelium leading to vaginal opening when the first ovulation occurs. The lower level of $E_{2}$ can also be responsible for the delayed vaginal opening compared with the control group. Thus, the late development of follicles delays the time at which this critical mass of follicles is reached. This suggestion is also reinforced by the delayed capacity of stressed rats to maintain a regular estrus cycling activity after vaginal opening. We cannot discard, however, that reproductive hypothalamus development in utero could be affected by the gestational stress.

\section{Conclusion}

We demonstrate for the first time that gestational stress produces early changes in follicular development and long-term impairments in ovarian function that finally leads to delayed puberty. Prenatal stress also reduces the pool of primordial follicles, which is closely related to the onset of reproductive senescence (Adhikari \& Liu 2009). Although we studied several possible mechanisms that explain the alteration of physiological processes in the ovary by the time they occur, the mechanism that explains how stress can induce these changes in ovarian function has not been established. A compensatory programing effect on sympathetic nerve activity that decreases the development of ovarian sympathetic nerves by a neurotrophic-dependent mechanism could be one of the causes that merit additional exploration.

\section{Declaration of interest}

The authors declare that there is no conflict of interest that could be perceived as prejudicing the impartiality of the research reported.

\section{Funding}

This work was supported, in part, by Fondecyt grant number 1130049 (to H E Lara) and Conicyt grant for doctoral thesis number 2411061 (to R Barra) and from Deutsche Forschungsgemeinschaft DFG 1080/19-1 and 1080/17-1 (to A Mayerhofer) and Conicyt-PIA-DFG10 binational program.

\section{References}

Adhikari D \& Liu K 2009 Molecular mechanisms underlying the activation of mammalian primordial follicles. Endocrine Reviews 30 438-464. (doi:10.1210/er.2008-0048)

Chomczynski P \& Sacchi N 1987 Single-step method of RNA isolation by acid guanidinium thiocyanate-phenol-chloroform extraction. Analytical Biochemistry 162 156-159. (doi:10.1016/0003-2697(87)90021-2)
Chrousos GP 1998 Stressors, stress, and neuroendocrine integration of the adaptive response. The 1997 Hans Selye Memorial Lecture. Annals of the New York Academy of Sciences 851 311-335. (doi:10.1111/j.17496632.1998.tb09006.x)

Cruz G, Barra R, Gonzalez D, Sotomayor-Zarate R \& Lara HE 2012 Temporal window in which exposure to estradiol permanently modifies ovarian function causing polycystic ovary morphology in rats. Fertility and Sterility 98 1283-1290. (doi:10.1016/j.fertnstert.2012.07.1060)

DeManno DA, Cottom JE, Kline MP, Peters CA, Maizels ET \& HunzickerDunn M 1999 Follicle-stimulating hormone promotes histone H3 phosphorylation on serine-10. Molecular Endocrinology 13 91-105. (doi:10.1210/mend.13.1.0222)

Dissen GA, Hirshfield AN, Malamed S \& Ojeda SR 1995 Expression of neurotrophins and their receptors in the mammalian ovary is developmentally regulated: changes at the time of folliculogenesis. Endocrinology 136 4681-4692.

Dissen GA, Parrott JA, Skinner MK, Hill DF, Costa ME \& Ojeda SR 2000 Direct effects of nerve growth factor on thecal cells from antral ovarian follicles. Endocrinology 141 4736-4750.

Dissen GA, Romero C, Hirshfield AN \& Ojeda SR 2001 Nerve growth factor is required for early follicular development in the mammalian ovary. Endocrinology 142 2078-2086.

Dissen GA, Garcia-Rudaz C \& Ojeda SR 2009 Role of neurotrophic factors in early ovarian development. Seminars in Reproductive Medicine 27 24-31. (doi:10.1055/s-0028-1108007)

Dorfman M, Arancibia S, Fiedler JL \& Lara HE 2003 Chronic intermittent cold stress activates ovarian sympathetic nerves and modifies ovarian follicular development in the rat. Biology of Reproduction $\mathbf{6 8}$ 2038-2043. (doi:10.1095/biolreprod.102.008318)

Ebling FJ 2005 The neuroendocrine timing of puberty. Reproduction 129 675-683. (doi:10.1530/rep.1.00367)

Fauser BC, Tarlatzis BC, Rebar RW, Legro RS, Balen AH, Lobo R, Carmina E, Chang J, Yildiz BO, Laven JS et al. 2012 Consensus on women's health aspects of polycystic ovary syndrome (PCOS): the Amsterdam ESHRE/ASRM-Sponsored 3rd PCOS Consensus Workshop Group. Fertility and Sterility 97 28-38.e25. (doi:10.1016/j.fertnstert. 2011.09.024)

Fiedler J, Jara P, Luza S, Dorfman M, Grouselle D, Rage F, Lara HE \& Arancibia S 2006 Cold stress induces metabolic activation of thyrotrophin-releasing hormone-synthesising neurones in the magnocellular division of the hypothalamic paraventricular nucleus and concomitantly changes ovarian sympathetic activity parameters. Journal of Neuroendocrinology 18 367-376. (doi:10.1111/j.1365-2826.2006.01427.x)

Glover V, O'Connor TG \& O'Donnell K 2010 Prenatal stress and the programming of the HPA axis. Neuroscience and Biobehavioral Reviews 35 17-22. (doi:10.1016/j.neubiorev.2009.11.008)

Goldstein DS \& Kopin IJ 2008 Adrenomedullary, adrenocortical, and sympathoneural responses to stressors: a meta-analysis. Endocrine Regulations 42 111-119.

Greiner M, Paredes A, Araya V \& Lara HE 2005 Role of stress and sympathetic innervation in the development of polycystic ovary syndrome. Endocrine 28 319-324. (doi:10.1385/ENDO:28:3:319)

Jara P, Rage F, Dorfman M, Grouselle D, Barra R, Arancibia S \& Lara HE 2010 Cold-induced glutamate release in vivo from the magnocellular region of the paraventricular nucleus is involved in ovarian sympathetic activation. Journal of Neuroendocrinology 22 979-986. (doi:10.1111/ j.1365-2826.2010.02040.x)

Lara HE, Hill DF, Katz KH \& Ojeda SR 1990a The gene encoding nerve growth factor is expressed in the immature rat ovary: effect of denervation and hormonal treatment. Endocrinology 126 357-363. (doi:10.1210/ endo-126-1-357)

Lara HE, McDonald JK \& Ojeda SR $1990 \mathrm{~b}$ Involvement of nerve growth factor in female sexual development. Endocrinology 126 364-375. (doi:10.1210/endo-126-1-364)

Levi-Montalcini R 1987 The nerve growth factor 35 years later. Science $\mathbf{2 3 7}$ 1154-1162. (doi:10.1126/science.3306916)

Mayerhofer A, Dissen GA, Costa ME \& Ojeda SR 1997 A role for neurotransmitters in early follicular development: induction of functional follicle-stimulating hormone receptors in newly formed follicles of the rat ovary. Endocrinology 138 3320-3329. 
Nugent BM, Tobet SA, Lara HE, Lucion AB, Wilson ME, Recabarren SE \& Paredes AH 2012 Hormonal programming across the lifespan. Hormone and Metabolic Research 44 577-586. (doi:10.1055/s-0032-1312593)

Pacak K, Baffi JS, Kvetnansky R, Goldstein DS \& Palkovits M 1998a Stressorspecific activation of catecholaminergic systems: implications for stressrelated hypothalamic-pituitary-adrenocortical responses. Advances in Pharmacology 42 561-564.

Pacak K, Palkovits M, Yadid G, Kvetnansky R, Kopin IJ \& Goldstein DS $1998 b$ Heterogeneous neurochemical responses to different stressors: a test of Selye's doctrine of nonspecificity. American Journal of Physiology 275 R1247-R1255.

Paredes A, Galvez A, Leyton V, Aravena G, Fiedler JL, Bustamante D \& Lara HE 1998 Stress promotes development of ovarian cysts in rats: the possible role of sympathetic nerve activation. Endocrine 8 309-315. (doi:10.1385/ENDO:8:3:309)

Pepling ME 2012 Follicular assembly: mechanisms of action. Reproduction 143 139-149. (doi:10.1530/REP-11-0299)

Pradelles P, Grassi J, Chabardes D \& Guiso N 1989 Enzyme immunoassays of adenosine cyclic $3^{\prime}, 5^{\prime}$-monophosphate and guanosine cyclic $3^{\prime}, 5^{\prime}$ monophosphate using acetylcholinesterase. Analytical Chemistry 61 447-453. (doi:10.1021/ac00180a014)

Ricu M, Paredes A, Greiner M, Ojeda SR \& Lara HE 2008 Functional development of the ovarian noradrenergic innervation. Endocrinology 149 50-56. (doi:10.1210/en.2007-1204)

Rinaudo P \& Wang E 2012 Fetal programming and metabolic syndrome. Annual Review of Physiology 74 107-130. (doi:10.1146/annurevphysiol-020911-153245)

Romero C, Paredes A, Dissen GA \& Ojeda SR 2002 Nerve growth facto induces the expression of functional FSH receptors in newly formed follicles of the rat ovary. Endocrinology 143 1485-1494. (doi:10.1210/ endo.143.4.8711)

Schobel HP, Fischer T, Heuszer K, Geiger H \& Schmieder RE 1996 Preeclampsia - a state of sympathetic overactivity. New England Journal of Medicine 335 1480-1485. (doi:10.1056/NEJM199611143352002)
Sirotkin AV 2011 Growth factors controlling ovarian functions. Journal of Cellular Physiology 226 2222-2225. (doi:10.1002/jcp.22588)

Sotomayor-Zarate R, Dorfman M, Paredes A \& Lara HE 2008 Neonatal exposure to estradiol valerate programs ovarian sympathetic innervation and follicular development in the adult rat. Biology of Reproduction $\mathbf{7 8}$ 673-680. (doi:10.1095/biolreprod.107.063974)

Sotomayor-Zarate R, Tiszavari M, Cruz G \& Lara HE 2011 Neonatal exposure to single doses of estradiol or testosterone programs ovarian follicular development-modified hypothalamic neurotransmitters and causes polycystic ovary during adulthood in the rat. Fertility and Sterility 96 1490-1496. (doi:10.1016/j.fertnstert.2011.09.011)

Szabo S 1998 Hans Selye and the development of the stress concept. Special reference to gastroduodenal ulcerogenesis. Annals of the New York Academy of Sciences 851 19-27. (doi:10.1111/j.1749-6632.1998. tb08972.x)

Tache Y \& Brunnhuber S 2008 From Hans Selye's discovery of biological stress to the identification of corticotropin-releasing factor signaling pathways: implication in stress-related functional bowel diseases. Annals of the New York Academy of Sciences 1148 29-41. (doi:10.1196/annals. 1410.007)

Takiuti NH, Kahhale S \& Zugaib M 2003 Stress-related preeclampsia: an evolutionary maladaptation in exaggerated stress during pregnancy? Medical Hypotheses 60 328-331. (doi:10.1016/S0306-9877(02) 00393-6)

Received 14 March 2014

First decision 9 April 2014

Revised manuscript received 1 May 2014

Accepted 7 May 2014 